\title{
Les systèmes de contrôle de la douleur
}

Ce deuxième article sur la douleur est centré sur la transmission des messages nociceptifs et leur modulation par des systèmes de contrôle locaux ou issus du tronc cérébral.

\section{Jean-Marie Besson}

Directeur de recherche au Cnrs et de l'unité de neurophysiologie pharmacologique, Inserm U I6I.

\section{REFERENCES}

I. Handwerker HO, Iggo A, Zimmermann M. Segmental and supraspinal actions on dorsal horn neurons responding to noxious and non noxious skin stimuli. Pain 1975; I: 147-65.

2. Melzack R, Wall PD. Pain mechanisms. A new theory. Science 1965; 150: 971-9.

3. Dubner R, Bennett GJ. Spinal and trigeminal mechanisms of nociception. Ann Rev Neurosci 1983; 6: 381-418.

\section{ADRESSE}

J.-M. Besson : Inserm U I6I, 2, rue d'Alésia, 75014 Paris.

$m / s n^{\circ}$ 9, vol. 2, novembre 86

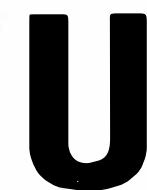

n premier article $\left(m / s n^{\circ} 6\right.$, vol. I, p. 306) a été consacré aux voies, relais et centres d'intégration des messages nociceptifs, mais c'est indiscutablement la mise en évidence des divers systèmes de contrôles qui constitue l'acquisition la plus importante dans le domaine de la physiologie de la douleur, au cours de ces dernières années.

Ces systèmes de contrôles ont surtout été étudiés à l'étage spinal où ils modulent la transmission des messages nociceptifs au niveau de la corne dorsale de la moelle qui ne peut donc plus être considérée comme un simple connecteur entre les nerfs périphériques et le cerveau. Comme nous le verrons, ces recherches ont eu des répercussions importantes pour le traitement des douleurs rebelles. C'est ainsi que différentes techniques neurochirurgicales qui consistaient à interrompre ou à détruire les voies et les relais de la douleur (section de nerfs, section de racines dorsales, cordotomie, lésions thalamiques...) ont tendance à être progressivement abandonnées. Dans toute la mesure du possible, ces techniques de neurochirurgie destructrice font place à des techniques de neurostimulation qui ont pour but de renforcer l'activité des systèmes de contrôles inhibiteurs et qui ont l'avantage de ne pas entraîner de lésions irréversibles du système nerveux.

Au niveau des relais spinaux, la transmission des messages nocicep- tifs est soumise à des systèmes de contrôle d'origine segmentaire et supraspinale.

\section{Les contrôles segmentaires}

Il a été clairement démontré que l'activation des fibres cutanées larges $(A \alpha, \beta)$, qui donne naissance à des sensations tactiles légères, bloque, au niveau médullaire, les réponses des neurones spinaux à des stimulations nociceptives. Cet effet inhibiteur qui s'exerce sur les neurones " nociceptifs " à l'origine des principaux faisceaux médullaires ascendants peut aussi être obtenu par la stimulation des colonnes dorsales [I] (figures $I$ et 2, pages 494 et 495). Dans ce dernier cas, l'inhibition résulte avant tout de la mise en jeu des collatérales que les fibres afférentes primaires des cordons postérieurs envoient à la substance grise médullaire ( figure 2). De fait, ces effets subsistent après une section spinale totale sus-jacente à la stimulation.

Ces données expérimentales expliquent en partie les effets favorables de l'utilisation thérapeutique des techniques de neurostimulation périphérique de faible intensité et de fréquence élevée. Dans ce cas, la stimulation peut être délivrée soit au niveau des nerfs périphériques par application d'électrodes sur la peau en regard du nerf ou par implantation chirurgicale si le nerf est profond, soit au niveau des cordons postérieurs de la moelle par 


\section{REFERENCES}

4. Sjölund BH, Eriksson MBE. The influence of Naloxone on analgesia produced by peripheral conditioning stimulation. Brain Res 1979; 173: 295-301.

5. Lamotte C, Pert CB, Synder SH. Opiate receptor binding in primate spinal cord. Distribution and changes after dorsal root section. Brain Res 1976; 11 2: 407-1 2.

6. Le Bars D, Guilbaud G, Jurna I, Besson JM. Differential effects of morphine on response of dorsal horn lamina $\mathbf{V}$ type cells elicited by $\mathbf{A}$ and C fibre stimulation in the spinal cat. Brain Res 1976; 1 1 3: 293-310.

7. Jessel TM, Iversen LL. Opiate analgesia inhibit substance $P$ release from rat trigeminal nucleus. Nature 1977; 268: 549-51.

8. Ruda MA. Opiates and pain pathways: demonstration of enkephalin synapses on dorsal projection neurones. Science 1 982; 2 I 5: 1 523-5

9. Yaksh TL. Spinal opiate analgesia: characteristics and principles of action. Pain $198 \mathrm{I}$; $1 \mathrm{I}$ : 293-346.

10. Besson JM, Lazorthes Y. Substances opiödes médullaires et analgésie (Spinal opioids and the relief of pain). Colloque Inserm, Vol 127. Paris: les éditions Inserm, 1985.

II. Yaksh TL, Harty GJ,. Effects of thiorphan on the antinociceptive actions of intrathecal (D-Ala ${ }^{2}$, Met $^{5}$ ) Enkephalin. Eur 7 Pharmacol 1982; 79: 293-300.

12. Terenius L, Nyberg F, Yaksh TL. Opioid peptides in the CNS. In: Besson JM, Lazorthes Y, eds. Substances opioides médullaires et analgésie (Spinal opioids and the relief of pain). Colloque Inserm, Vol 127. Paris : les éditions Inserm, 1985:317-32.

13. Cesselin F. Montastruc JL, Gros C, Bourgoin S, Hamon $M$. Met-Enkephalin levels and opiate receptors in the spinal cord of chronic suffering rats. Brain Res 1980; 191: 289-93.

14. Cesselin F, Bourgoin S, Artaud F, et al. The release of Met-Enkephalin-like material at the spinal level: in vivo and in vitro studies. In: Besson JM, Lazorthes Y, eds. Substances opioides médullaires et Analgésie (Spinal opioids and the relief of pain). Colloque Inserm, Vol 127. Paris : les éditions Inserm, 1985; 241-64

15. Roques BP. Pharmacologie moléculaire des neuropeptides. médecine/sciences 1986; $2: 17-25$.

16. Willer JC, Bussel B. Evidence for a direct spinal mechanism in morphine-induced inhibition of nociceptive reflexes in humans. Brain Res 1980; 1 87: 21 2-5.

17. Yaksh TL, Elde RP. Factors governing release of methionin enkephalin-like immunoreactivity from mesencephalon and spinal cord of the cat in vivo. 7 Neurophysiol $1981 ; 46$ : 1056-75.

18. Reynolds DV. Surgery in the rat during electrical analgesia induced by focal brain stimulation. Science 1969; 164: 444-5.

A1

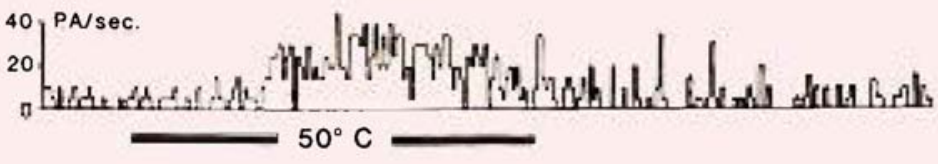

A2

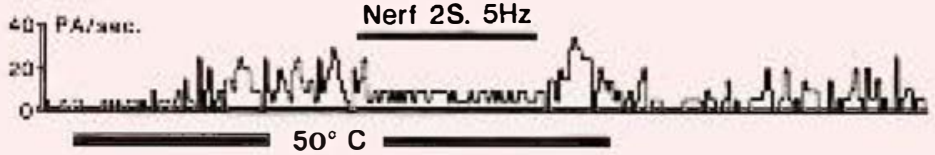

B1

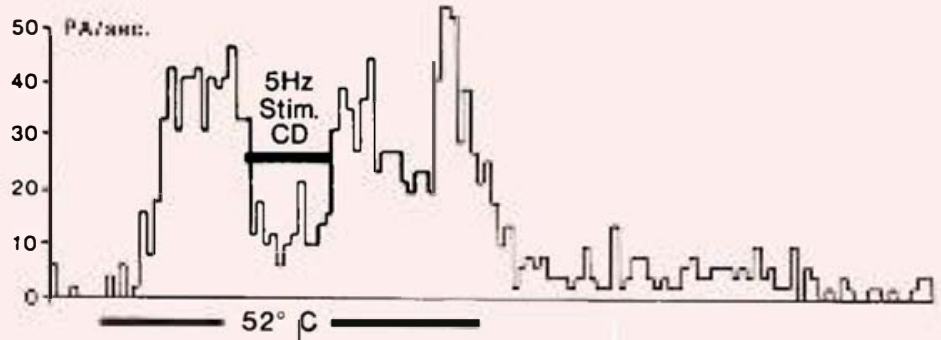

$\mathrm{B} 2$

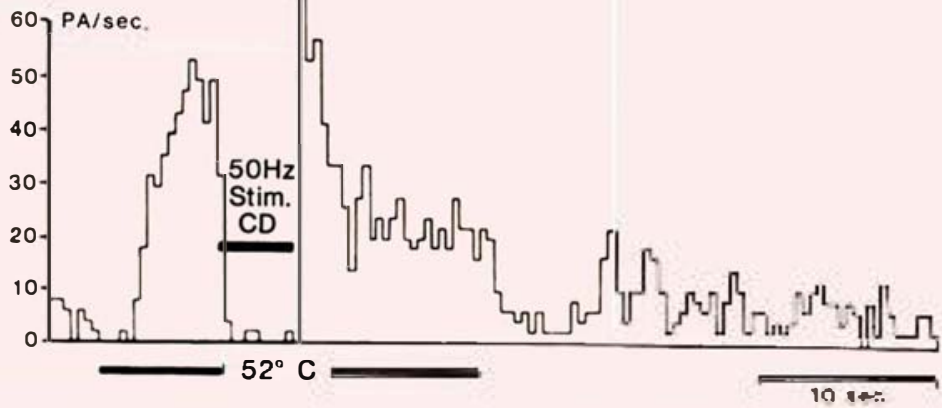

Figure 1. Inhibition segmentaire de neurones nociceptifs de la corne dorsale de la moelle enregistrée au niveau lombaire.

A. La réponse induite par la chaleur nociceptive $\left(A_{1}, 50^{\circ} \mathrm{C}\right)$ est inhibée par la stimulation des fibres de gros calibre du nerf plantaire $\left(A_{2}\right)$.

$B$. Les réponses à la chaleur nociceptive $\left(52^{\circ} \mathrm{C}\right) d^{\prime}$ un autre neurone sont inhibées par la stimulation des colonnes dorsales (CD), fréquences de $5 \mathrm{~Hz}\left(B_{1}\right)$ et $50 \mathrm{~Hz}$ (B2) (d'après [1]).

implantation d'électrodes en position extradurale par voie percutanée. Ces techniques de neurostimulation se sont surtout révélées efficaces dans le cas des douleurs de déafférentation*

Cependant, une discordance importante entre la clinique et l'expérimentation est à mentionner : alors que les patients sont soulagés pendant des heures après quelques minutes de stimulation, l'inhibition neuronale observée au niveau médullaire chez l'animal normal

- Déafférentation : déconnection partielle ou totale (chirurgicale, traumatique ou pathologique) d'une structure nerveuse d'avec les systèmes de fibres afférentes. cesse pratiquement dès l'arrêt de la stimulation. Cette discordance, difficile à expliquer, pourrait résulter de la différence de réactivité des mécanismes neuronaux impliqués dans la nociception chez l'animal normal et dans les douleurs chroniques chez l'homme.

Du point de vue électrophysiologique, l'existence de mécanismes d'inhibition pré et/ou postsynaptique a été proposée pour expliquer les effets inhibiteurs observés sur les neurones spinaux nociceptifs, lors de l'activation de fibres cutanées de grand diamètre; l'intervention de la substance gélatineuse (couche 2 de la corne dorsale) a été initialement 


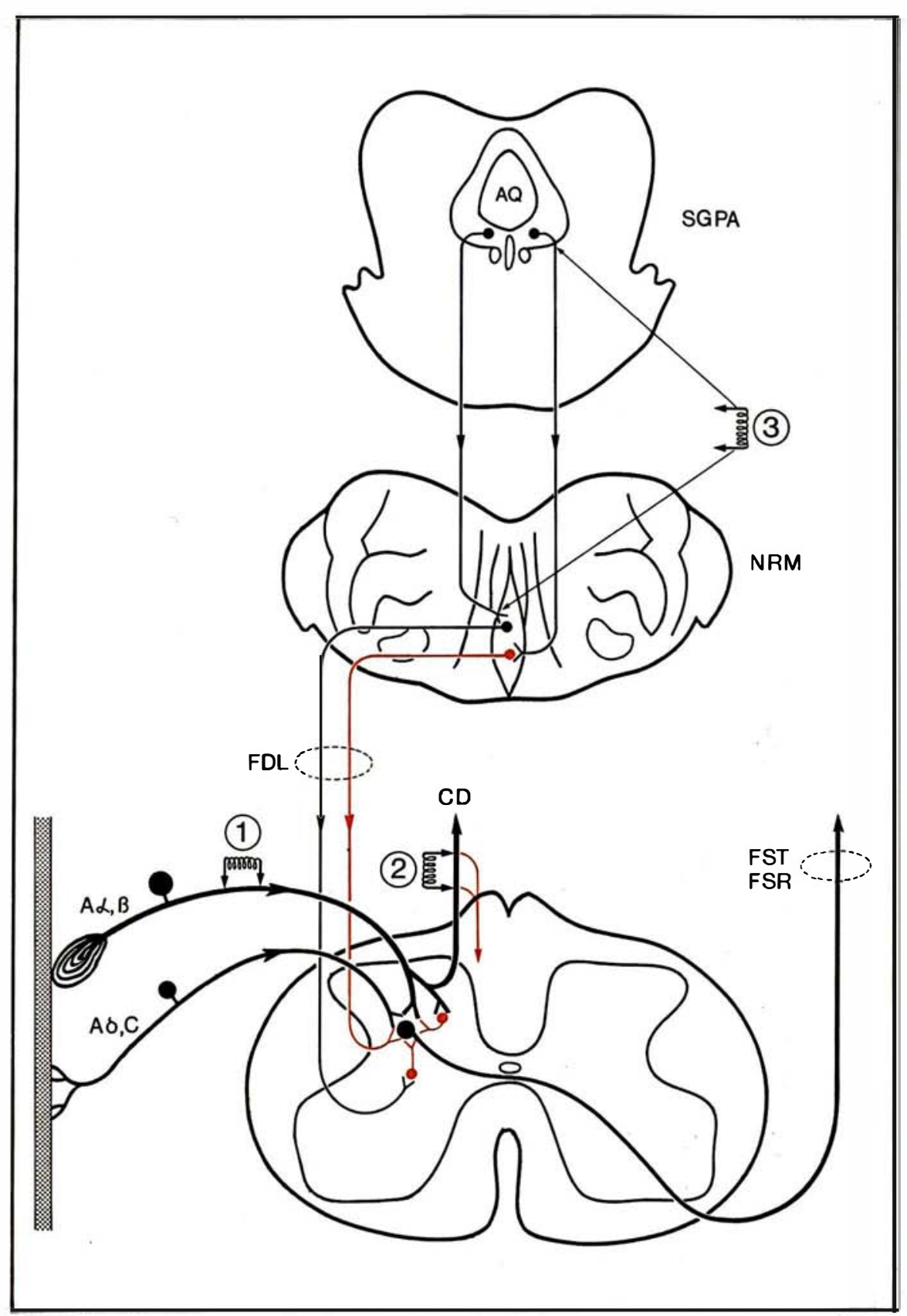

Figure 2. Contrôles de la transmission des messages nociceptifs au niveau médullaire. Les messages nociceptifs véhiculés par les fibres $A \delta$ et $C$ activent les neurones de la corne dorsale dont les axones croisent la ligne médiane et se projettent vers les centres supérieurs par l'intermédiaire des faisceaux spinothalamiques (FST) et spinoréticulaire (FSR). Cette transmission peut être modulée au niveau segmentaire : (1) par la stimulation, entre la périphérie et la moelle, des fibres afférentes de gros calibre $(A \alpha, \beta)$ dont l'activation donne naissance à des sensations tactiles légères; (2) par la stimulation des fibres de gros diamètre des colonnes dorsales (CD). Dans ce cas, il s'agit d'une activation antidromique de l'axone (flèche rouge): (3) les contrôles d'origine supraspinale s'exercent à partir de la substance grise périaqueducale (SGPA) ou du noyau raphé Magnus (NRM). Les fibres descendantes en provenance de cette dernière région sont localisées dans le faisceau dorsolatéral de la moelle (FDL). $A Q=a-$ queduc. Ces différents types de contrôles peuvent s'exercer soit directement au niveau du corps cellulaire des neurones nociceptifs, soit par l'intermédiaire d'interneurones inhibiteurs qui sont figurés en rouge.

$m / s n^{\circ}$ 9, vol. 2, novembre 86 suggérée [2] mais son rôle reste mal connu malgré des études récentes qui ont réussi à analyser les caractéristiques électrophysiologiques, neurochimiques et anatomiques des neurones de cette région (voir références dans [3]).

Du point de vue pharmacologique, la substance gélatineuse et la couche marginale de la corne dorsale contiennent de nombreux composés : peptides dans les terminaisons des fibres afférentes, peptides (notamment enképhaline et dynorphine...) et acide gamma aminobutyrique dans certains neurones intrinsèques, monoamines et peptides dans les terminaisons de certaines fibres descendantes issues du tronc cérébral. L'implication des endomorphines médullaires (enképhalines et dynorphine) ne semble pas être démontrée pour expliquer les effets de la stimulation des grosses fibres puisqu'aussi bien les effets inhibiteurs obtenus chez l'animal que l'hypoalgésie observée chez l'homme ne sont pas modifiés après administration d'un antagoniste de la morphine (naloxone) [4].

\section{Action spinale de la morphine}

La présence de récepteurs opioïdes sur les terminaisons des fibres afférentes [5] suggère que les substances morphiniques peuvent dans d'autres circonstances avoir des effets présynaptiques sur la transmission des messages nociceptifs au niveau spinal. Ceci est en bon accord avec le fait que chez l'animal dont la moelle a été déconnectée des structures supraspinales, l'administration intraveineuse de morphine déprime très puissamment les réponses des neurones de la corne dorsale à des stimulations nociceptives. Ces effets dépendant de la dose, stéréospécifiques, renversés par la naloxone, s'exercent préférentiellement sur les réponses dues à l'activation des fibres fines $\mathrm{A} \delta$ et $\mathrm{C}[6]$ (figure 3 , p. 496). Il a été proposé que les effets de la morphine s'exerceraient au niveau présynaptique en bloquant la libération de substance $P$ (SP) qui serait le neurotransmetteur libéré par les terminaisons des fibres fines [7], (figure 4, p. 497). Cependant le rôle de la substance $P$ est 
actuellement très controversé et l'existence de synapses enképhalinergiques axo-axoniques au niveau des fibres afférentes primaires n'a pas été établie. En revanche, des études immunohistochimiques ont clairement identifié des terminaisons enképhalinergiques en position présynaptique par rapport aux neurones médullaires à l'origine des principaux faisceaux ascendants [8], (figure 4).

Quel que soit le mécanisme intime des modalités de l'action spinale de la morphine, les études comportementales effectuées chez l'animal [9] ont clairement établi que l'administration intrathécale de cette substance à faibles doses provoquait des effets analgésiques puissants, durables et localisés au niveau des segments sous-jacents à l'injection. L'ensemble de ces données a conduit à l'utilisation de ce mode d'administration pour le traitement de certaines douleurs rebelles chez l'homme (références dans [Io]). L'administration intrarachidienne de morphine qui s'effectue par l'intermédiaire de systèmes implantables (réservoirs sous-cutanés) constitue une alternative efficace aux autres thérapeutiques et notamment à la chirurgie d'interruption des voies de la nociception; elle s'adresse préférentiellement aux douleurs rebelles d'origine néoplasique. Les objectifs de cette thérapeutique séduisante par son efficacité, sa sélectivité, son caractère peu invasif et conservateur sont aussi d'ordre socio-économique : (a) Confort du patient, que la médecine ne peut plus sauver mais qu'elle a maintenant les moyens de soulager efficacement de ces douleurs autrefois irréductibles. (b) Réduction de l'hospitalisation : possibilité d'effectuer ce traitement en ambulatoire, permettant ainsi à ces patients atteints de cancers avancés de regagner leur domicile et de terminer le plus "confortablement" possible leur vie en famille.

\section{Système opioïde endogène spinal}

Les couches superficielles de la corne dorsale (zone marginale et substance gélatineuse) sont riches en récepteurs opioïdes et en termi- naisons et corps cellulaires enképhalinergiques. L'existence à ce niveau d'un système de contrôle endomorphinique endogène a donc été suggérée. La morphine agirait au niveau spinal en mimant et renforçant un mécanisme physiologique, normalement assuré par les enképhalines. Cependant, il est difficile d'apprécier l'activité d'un tel système. C'est ainsi que l'administration intrathécale de thiorphan (inhibiteur d'enképhalinase) n'affecte pas diverses réactions nocicep- tives mais est cependant capable de potentialiser l'effet d'un dérivé de l'enképhaline [II]. Du point de vue biochimique, les données sont décevantes car les différents groupes [12] n'ont pas pu mettre en évidence chez l'homme une corrélation entre la concentration d'endomorphine dans le liquide céphalo-rachidien (LCR)-et le seuil de perception de la douleur ou la présence de douleurs chroniques. En revanche, dans un modèle de douleur expérimentale (rat arthri-

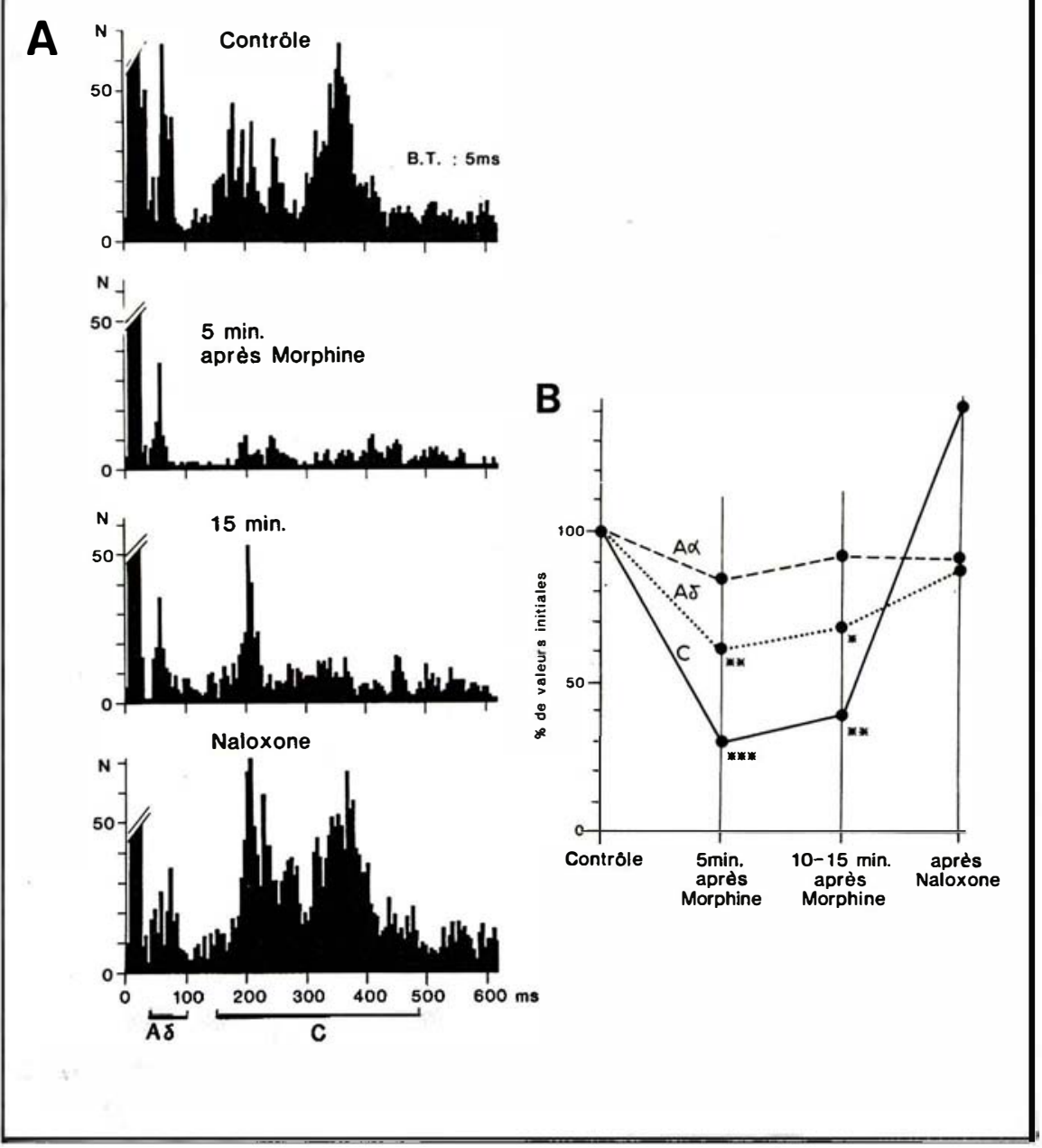

Figure 3. A. Action dépressive directe de la morphine $(2 \mathrm{mg} / \mathrm{kg} / \mathrm{iv})$ sur les réponses d'un neurone de la corne dorsale de la moelle à des stimulations électriques intenses du nerf sural. Cette substance, dont les effets sont renversés par la naloxone $(0.2 \mathrm{mg} / \mathrm{kg} / \mathrm{iv})$, déprime de façon importante les réponses cellulaires dues à l'activation des fibres $C$ et $A \delta$. B. Résultats globaux (15 neurones) montrant que la morphine n'affecte pas les réponses dues à l'activation des grosses fibres cutanées $(A \alpha, \beta)$ mais que ses effets s'exercent préférentiellement sur celles dues à la mise en jeu des fibres fines (C et $A \delta$ ) (d'après [6]). 
tique), une augmentation des concentrations tissulaires en metenképhaline [13] a été observée au niveau de la moelle dorsale. Paradoxalement, sur ce modèle, la libération spontanée de met-enképhaline mesurée in vivo au niveau du LCR, est considérablement réduite par rapport à celle observée chez l'animal normal [I4].

Une autre approche pour évaluer l'activité du système opioïde endogène spinal, a consisté à utiliser l'administration d'un antagoniste (la naloxone) qui, tout en ayant une préférence marquée pour les récepteurs mu [15], est capable à des doses modérément élevées, de se fixer sur l'ensemble des récepteurs opioïdes. Chez l'animal spinal, les eff ets de la naloxone sont loin d'être univoques puisque, si des effets facilitateurs sur les réponses des neurones de la corne dorsale ou sur certains réflexes nociceptifs ont été rapportés par certains auteurs, de nombreux autres n'ont pas observé de modification. De même chez des patients paraplégiques, la naloxone ne facilite pas un réflexe spinal nociceptif [16].

En clinique, nous avons déjà signalé que les effets analgésiques induits par les techniques de neurostimulation périphérique de faible intensité et de fréquence élevée, qui

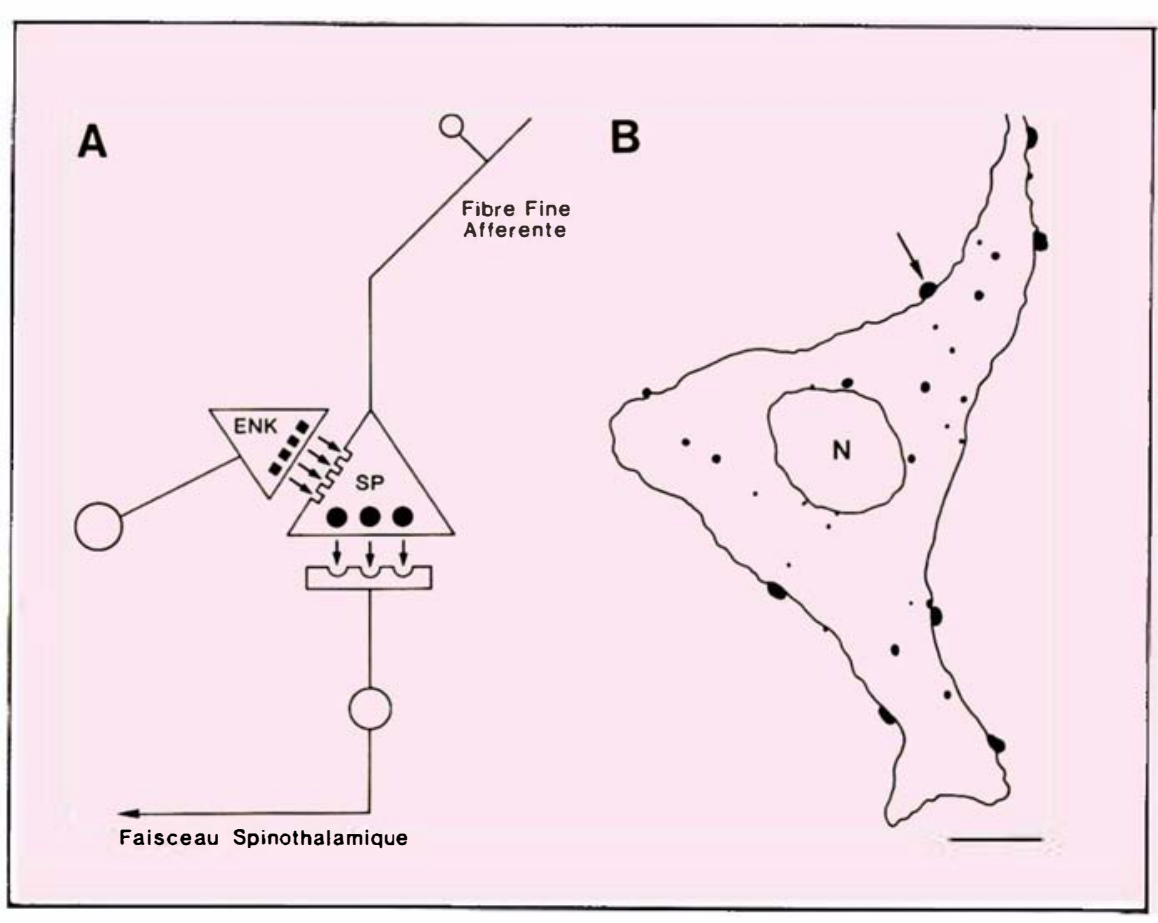

Figure 4. Modalités d'action de la morphine sur la transmission des messages nociceptifs au niveau spinal. A. Le neurotransmetteur libéré par les afférences de fin calibre serait la substance $P(S P)$ qui excite les neurones de la corne dorsale. Certains récepteurs morphiniques ( $\sim \sim \Omega$ ) se trouvent sur ces afférences car leur taux est considérablement diminué après rhizotomie dorsale. La morphine ou les enképhalines (ENK-synapse axoaxonique) agiraient au niveau présynaptique en bloquant la libération de SP (d'après [7]). Cependant, la démonstration anatomique de l'existence de synapses enképhalinergiques axo-axoniques sur les terminaisons des fibres afférentes primaires reste à faire. B. En revanche, l'existence de terminaisons enképhalinergiques axo-somatiques sur des neurones de la corne dorsale à l'origine du faisceau spinothalamique a été clairement établie. II s'agit de la reconstruction du soma d'un neurone spinothalamique ( $N$ : Noyau). La flèche indique la présence d'une terminaison enképhalinergique. Les grains noirs à l'intérieur du soma révèlent la présence de péroxydase du raifort qui a été captée par les terminaisons de ce neurone au niveau thalamique et a migré de façon rétrograde vers les neurones de la corne dorsale (d'après [8]). activent les fibres de gros calibre, n'étaient pas sous-tendus par des mécanismes endomorphiniques. En revanche, l'analgésie induite par des stimulations périphériques de basse fréquence et d'intensité élevée qui activent vraisemblablement les fibres fines, semble être associée à une libération de substances opioïdes endogènes [4]. Cette observation est en bon accord avec le fait que chez l'animal spinal, une augmentation de la libération de metenképhaline dans le liquide de superfusion de la moelle épinière a été mise en évidence après stimulation des fibres fines $A \delta$ et $C$ [ 17$]$ ou après stimulation thermique intense [14]. Cependant, chez l'animal intact, la libération d'enképhaline induite au niveau spinal par des stimulations nociceptives peut également résulter de l'activation d'une boucle supraspinale mettant en jeu les systèmes descendants bulbospinaux [14]. Par sa complexité (multiplicité des récepteurs opioïdes et des endomorphines...), l'étude des systèmes endogènes spinaux reste donc difficile à aborder mais son implication fonctionnelle est bien illustrée par les effets analgésiques puissants et de longue durée provoqués par l'administration intrathécale de morphine.

\section{Contrôles d'origine supraspinale}

Ils s'exercent principalement-à partir de certaines régions du tronc cérébral dont les neurones sont à l'origine de voies descendantes inhibitrices. Initialement il a été mis en évidence, chez le rat, que la stimulation de la substance grise périaqueducale (SGPA) entraîne des effets analgésiques importants [18]. Lors d'une étude extensive, chez le chat (figure 5, p. 498), il a été précisé que ces effets ont principalement pour origine la région ventrale de la SGPA qui correspond au noyau dorsal du raphé riche en corps cellulaires sérotoninergiques [19]. Ces auteurs ont confirmé le rôle d'autres noyaux du raphé, notamment au niveau du pont et du bulbe, où des effets analgésiques extrêmement puissants ont été déclenchés par la stimulation du noyau raphé Magnus (NRM) 


\section{REFERENCES}

19. Oliveras JL, Besson JM, Guilbaud G, Liebeskind JC. Behavioral and electrophysiological evidence of pain inhibition from brain stimulation in the cat. Exp Brain Res 1974; 20: 32-44.

20. Basbaum AI, Fields HL. Endogenous pain control systems: brainstem spinal pathways and endorphin circuitry. Ann Rev Neurosci 1984; 7: 309-38.

2I. Basbaum AI, Clanton CH, Fields HL. Opiate and stimulus produced analgesia: functional anatomy of a medullo-spinal pathway. Proc Nat Acad $S c i(U S A)$ 1976; 73: 4685-8.

22. Oliveras JL, Hosobuchi Y, Guilbaud G, Besson JM. Analgesic electrical stimulation of the feline nucleus raphè magnus: development of tolerance and its reversal by 5-HTP. Brain Res 1978; 146: 404-9.

23. Akil H, Mayer DJ, Liebeskind JC. Antagonism of stimulation produced analgesia by naloxone, a narcotic antagonist. Science 1976; 191: 96I-2.

24. Oliveras JL, Hosobuchi Y, Redjemi F, Guilbaud G, Besson JM. Opiate antagonist nalaxone strongly reduces analgesia induced by stimulation of the raphe nucleus (centrales inferior) Brain Res I 977; 120 : 22I -9.

25. Dionne RA, Mueller GP, Young RF, et al. Contrast medium causes the apparent increase in B-endorphin levels in human cerebrospinal fluid following brain stimulation. Pain I984; 20: 313 21.

26. Fessler RG, Brown FD, Rachlin JR, Mullan $S$, Fang VS. Elevated $\beta$-endorphin in cerebrospinal fluid after electrical brain stimulation: artefact of contrast infusion? Science 1984; 224: 1017-9.

27. Le Bars D, Dickenson AH, Besson JM. Diffuse noxious inhibitory controls (DNIC). I effects on dorsal horn convergent neurons in the rat. Pain 1979; 6: 283-304

28. Le Bars D, Dickenson AH, Besson JM. The triggering of bulbo-spinal serotoninergic inhibitory controls by noxious peripherical inputs. In Sjölund B, Björklund A, eds. Brain stem control of spinal mechanism. Amsterdam: Elsevier Biomedical Press, 1982: 38I-4Io.

29. Le Bars D, Dickenson AH, Besson JM Opiate analgesia and descending control systems In: Bonica JJ, Lindblom U, Iggo A, eds. Advances in pain research and therapy. New York: Raven Press, 1983; 5: 34I-72.

30. Fardin V, Oliveras JL, Besson JM. A reinvestigation of the analgesic effects induced by stimulation of the periaqueductal gray matter in the rat. I. The production of behavioural side effects together with analgesia. Brain Res 1984; 366: I 05-23.

31. Mazars G, Roge R, Mazars Y. Résultats de la stimulation du faisceau spinothalamique et leur incidence sur la physiopathologie de la douleur. Rev Neurol (Paris) 1960; 136-8.
$I_{A}$

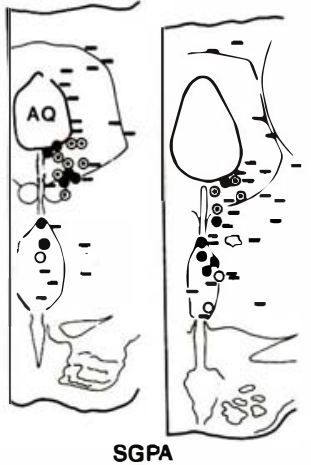

$\Pi_{\mathrm{A}}$
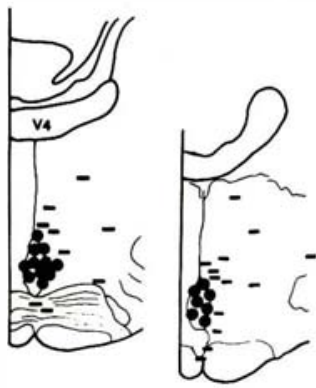

NRM

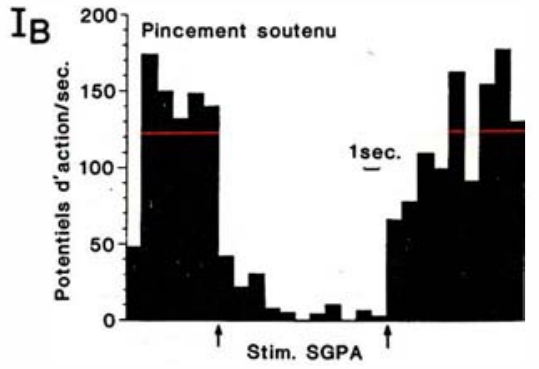

$\Pi_{B}$
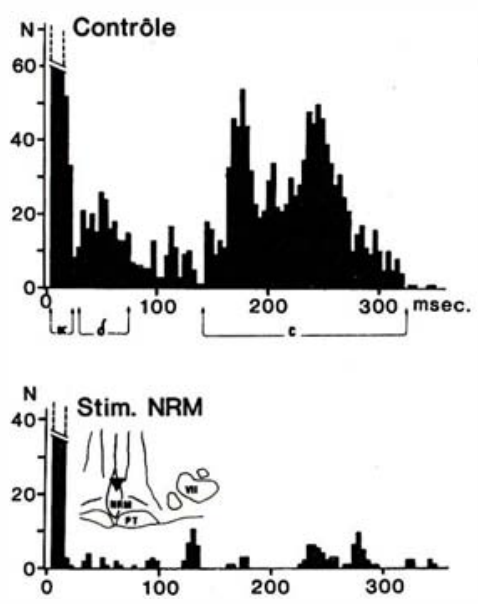

Figure 5. Effets induits par les stimulations électriques du tronc cérébral. $A Q=$ aqueduc; $V 4=4^{e}$ ventricule. Les sites dont la stimulation induit des effets analgésiques sont indiqués par des cercles noirs, l'absence d'effet par des tirets noirs.

1. Coupe frontale au niveau de la substance grise périaqueducale (SGPA). A. Localisation des sites dont la stimulation induit des effets analgésiques chez le chat libre de ses mouvements. $B$. Inhibition de la réponse au pincement d'un neurone nociceptif lors de la stimulation de la SGPA chez le chat anesthésié (d'après [19]).

II. Coupe frontale au niveau du noyau raphé magnus (NRM). A. Localisation des sites de stimulation induisant des effets analgésiques chez le chat libre de ses mouvements. B. Effets inhibiteurs induits par stimulation du NRM sur la réponse d'un neurone nociceptif non spécifique à la stimulation électrique transcutanée. Notez que les réponses dues à l'activation des fibres $A \delta$ et $C$ sont totalement supprimées par cette stimulation centrale. (Modifié d'après [22, 24]).

( figure 5). Le fait que la stimulation de la SGPA bloque certains réflexes nociceptifs suggère que l'analgésie résulte, au moins en partie, de la mise en jeu de voies descendantes inhibitrices, ce qui est en bon accord avec la démonstration que la même stimulation déprime de façon intense les réponses des neurones de la corne dorsale à des stimulations nociceptives [19] (figure 5). Des résultats compa- rables ont été obtenus en stimulant le NRM. Du point de vue anatomique, il est clairement établi que les neurones de ce noyau se projettent- massivement au niveau de la corne dorsale. En revanche, les projections spinales directes à partir de la SGPA sont rares et l'on admet que cette région exerce principalement son action par l'intermédiaire du NRM [20]. Les effets analgésiques induits à partir de la SGPA 
sont d'ailleurs abolis en dessous d'une lésion bilatérale du faisceau dorsolatéral de la moelle qui contient de nombreux axones descendants, notamment sérotoninergiques, issus du NRM [2I].

La pharmacologie des systèmes descendants est très complexe, et elle est l'objet de multiples travaux qui sont souvent difficiles à interpréter car les méthodologies utilisées sonit très variées. Très schématiquemént, retenons que :

(a) la participation des voxes bulbospinales sérotoninergiques est bien acquise tant du peint de vue électrophysiologique que du point de vue comportemental. Mentionnons à ce sujet que l'épuisement des effets induits par des stimulations répétées de NRM (phénomène de toléran $r_{\text {:e) }}$ peut être corrigé par l'administration de précurseurs de la sérotonine [22];

(b) si de nombreuses données plaident en faveur de l'implication des systèmes descendants noradrénergiques, les études comportementales restent insuffisantes;

(c) il a été démontré que l'administration de naloxone (antagoniste des substances opioïdes) abolit ou réduit les eff ets analgésiques induits par stimulation de la SGPA [23] ou du NRM [24], ce qui suggère que la stimulation centrale entraîne une libération d'endomorphines. Ces résultats sont à rapprocher de données biochimiques montrant chez l'homme un accroissement d'analogues immunoréactifs de la $\beta$-endorphine et des enképhalines dans le LCR après stimulation de la SGPA. Cependant, des résultats récents suggèrent que l'augmentation de la concentration de $\beta$-endorphine dans le LCR aurait pour origine l'injection du produit de contraste, néces-

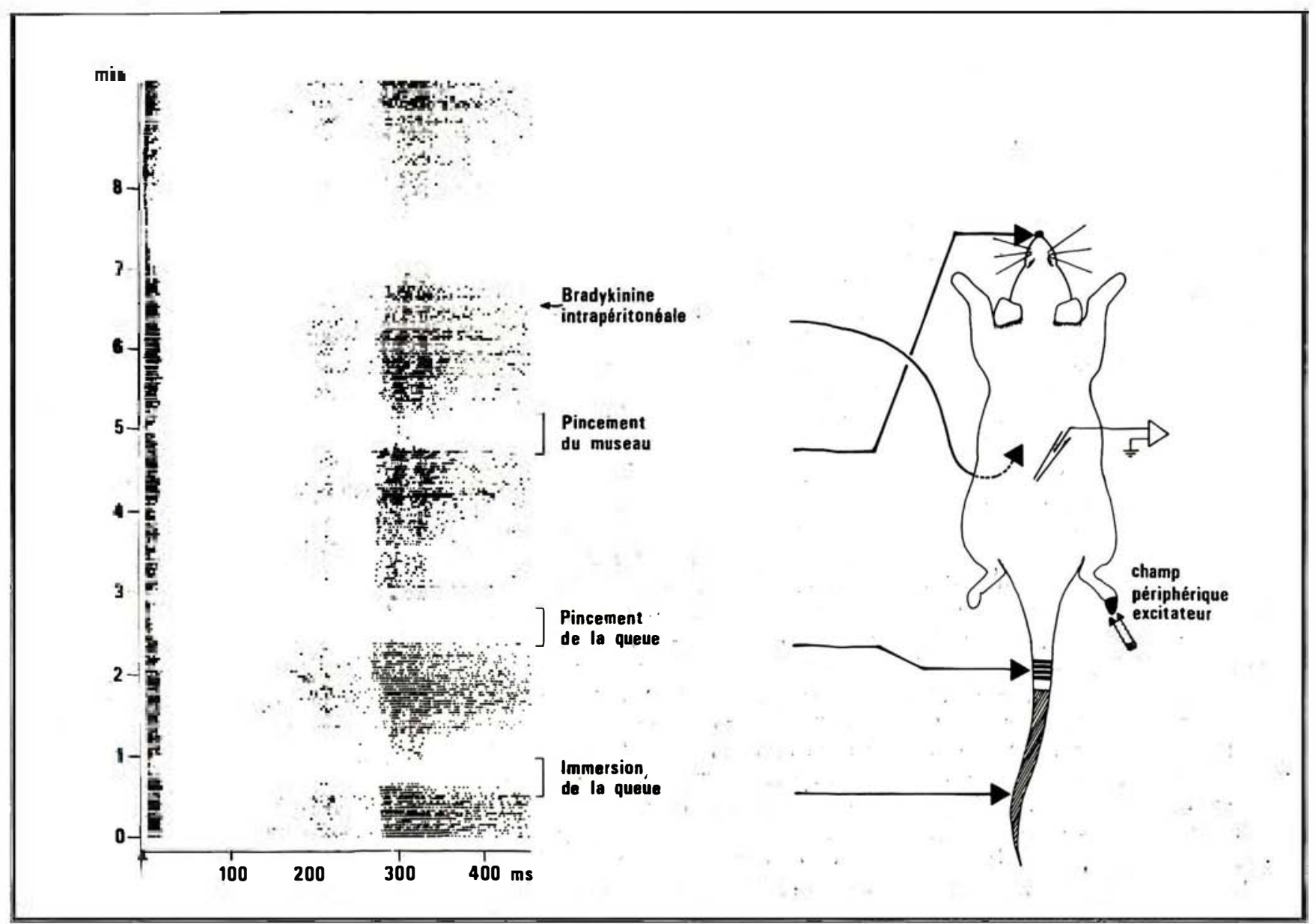

Figure 6. Mise en évidence des contrôles inhibiteurs diffus induits par stimulation nociceptive. Ces stimulations sont appliquées au niveau de différentes parties du corps de l'animal. On enregistre l'effet sur les réponses d'une cellule nociceptive non spécifique de la corne dorsale à la stimulation électrique intense de son champ excitateur périphérique. Dans ce type de représentation, chaque potentiel d'action est transformé en un point. Les réponses successives à la stimulation électrique transcutanée de l'extrémité de la patte sont superposées, l'origine de chaque stimulation étant placée sur la même verticale (flèche). Cette stimulation déclenche une double activation du neurone: l'une de brève latence $(5-7 \mathrm{~ms})$ due à l'activation des fibres $A$ et l'autre, de longue latence (300 ms environ) due à l'activation des fibres $C$. La lecture de bas en haut donne une évaluation qualitative de l'évolution de la réponse : on peut observer que les quatre stimulations nociceptives successivement appliquées sur la queue (immersion dans de l'eau à $52^{\circ} \mathrm{C}$, pincement). le museau (pincement) ou les viscères (injection intra-péritonéale de bradykinine) inhibent très puissamment les réponsẹs dues aux fibres $C$ et, dans une moindre mesure, celles dues aux fibres $A$ (d'après [27]). 
saire pour visualiser les électrodes, dans le système ventriculaire $[25,26]$. Étant donné les problèmes éthiques posés chez l'homme, ces études devront être reprises chez l'animal afin de démontrer une corrélation stricte entre les effets analgésiques et la libération de ces substances. D'autre part, la signification fonctionnelle de ces polypeptides dans le LCR devra, bien entendu, être précisée.

\section{Les systèmes descendants}

A partir de ces données, différents auteurs (références dans [20]) ont proposé l'existence d'un système analgésique endogène dans lequel la SGPA et le NRM feraient partie d'une boucle de rétroaction négative mise en jeu par des stimulations intenses et entraînant en retour l'inhibition de la transmission des messages nociceptifs au niveau médullaire. Les travaux relatifs aux contrôles inhibiteurs diffus induits par des stimulations nociceptives (CIDN) démontrent l'existence d'un tel système [27]. Dans ce cas, comme le montre la figure $6, p .499$, certains neurones nociceptifs de la corne dorsale (qui possèdent un champ excitateur périphérique bien localisé) peuvent être puissamment inhibés par des stimulations nociceptives appliquées sur n'importe quelle partie du corps de l'animal, à la condition qu'elle soit distincte du champ périphérique excitateur de ces neurones. Les CIDN qui sont en partie sous-tendus par des mécanismes sérotoninergiques dépendent de l'intégrité d'une boucle spino-bulbo-spinale dont la partie ascendante correspond principalement au faisceau spinothalamique, la partie descendante empruntant le faisceau dorsolatéral de la moelle [28].

Du point de vue clinique, les CIDN pourraient représenter le substrat fonctionnel permettant d'expliquer certaines observations paradoxales telles que le masquage d'une douleur par une douleur d'origine différente (contre irritation) [28]. Il en est vraisemblablement de même pour certaines formes d'analgésie obtenues par hyperstimulation ou par acupuncture, puisque certains auteurs précisent que les effets de ces stimulations sont d'autant plus efficaces qu'elles sont plus intenses, voire à la limite du supportable pour le sujet [28].

Sur la base de certaines analogies existant entre l'analgésie induite par stimulation cérébrale profonde et l'analgésie morphinique, il a été également suggéré que cette substance pouvait entraîner un renforcement de l'activité des contrôles inhibiteurs descendants [20]. Des injections de quelques microgrammes de morphine au niveau de la SGPA ou de NRM induisent des effets analgésiques. Néanmoins, la démonstration électrophysiologique d'un accroissement des contrôles inhibiteurs descendants par la morphine reste très controversée [29].

En clinique, la stimulation des régions périaqueducales et périventriculaires est utilisée pour le traitement des douleurs intenses chroniques rebelles. Les résultats de cette approche thérapeutique, qui a été pratiquée sur quelques centaines de patients, sont difficiles à évaluer même après un recul de dix ans. A partir de données obtenues chez l'animal [30], les divergences qui existent pourraient s'expliquer par des difficultés rencontrées en neurochirurgie stéréotaxique pour stimuler les zones efficaces qui semblent être bien localisées. Pour cette raison, bon nombre d'équipes neurochirurgicales qui prennent en charge les douleurs intraitables, semblent de nouveau avoir recours, notamment pour les douleurs de déafférentation, à la stimulation du noyau ventropostérolatéral du thalamus, telle qu'elle avait été préconisée dès les années I96o [3I].

En conclusion, la mise en évidence des systèmes de contrôles a permis d'établir de nouvelles approches dans la lutte contre la douleur, et une meilleure connaissance de la pharmacologie de ces systèmes devrait permettre de proposer de nouveaux traitements plus spécifiques et plus efficaces. Le rôle des endomorphines reste à préciser bien que ces substances existent dans des structures qui jouent un rôle stratégique notoire dans la nociception

\section{Summary}

The transmission of nociceptive messages at the spinal relay is under both segmental and supraspinal controls. Segmental controls are characteristically inhibitory effects produced by large diameter af ferent fibers on the response of spinal neurons to nociceptive stimulation. These esfects can partly explain the therapeutic effectiveness of transceltaneous electrical stimulation. Descending inhibitory controls : xre mainly originating from brainstem areas (periaqueductal gra y matter, ventromedial medullaj which directly or indirectly send projection at the level of the dorsal horn in order to modulate the transmission of noxious messages. Serotoninergic, noradrenergic and endogenous opioids mechanisms partly mediate the inhibitory effects. Despite the many gaps in our knowledge of the physiology and pharmacology of these control systems, their existence has had important consequences for the field of pain. Thus the various techniques used to interrupt or destroy the pathways or relays involved in pain have gradually been abandoned. These neurosurgical approaches are progressively being replaced by other techniques (neurostimulation, intrathecal morphine...) which aim at reinforcing the activity of inhibitory controls with the advantage of not producing irreversible lesions.

\section{TIRES A PART}

J.-M. Besson : Inserm U I61, 2, rue d'Alésia, 75014 Paris. 